Received 01/20/2020 Review began $02 / 18 / 2020$ Review ended 10/05/2020 Published 10/06/2020 Corrected 07/14/2021

(c) Copyright 2020

Shah et al. This is an open access article distributed under the terms of the Creative Commons Attribution License CC-BY 4.0. which permits unrestricted use, distribution, and reproduction in any medium, provided the original author and source are credited.

\section{Corrected: A Review of Non-Surgical Pain Management in Osteoarthritis}

\author{
Neha Shah ${ }^{1}$, Shaheen Jadidi ${ }^{2}$, Prempreet Bajaj ${ }^{3}$ \\ 1. Department of Physical Medicine and Rehabilitation, Northwestern Medicine at Marianjoy Rehabilitation Hospital, \\ Wheaton, USA 2. Family Medicine, McGaw Medical Center of Northwestern University, Chicago, USA 3. Department of \\ Orthopedic Surgery and Rehabilitation, Loyola University Medical Center, Maywood, USA
}

Corresponding author: Shaheen Jadidi, shaheen.jadidi@northwestern.edu

\section{This article has been corrected.}

Correction date: July 14, 2021. Cite this correction as Shah N, Jadidi S, Bajaj P (July 14, 2021) Correction: A Review of Non-Surgical Pain Management in Osteoarthritis. Cureus 13(7): c45. doi:10.7759/cureus.c45.

Due to a misunderstanding between authors, two of the original authors were not listed on this article at the time of submission and publication. All three authors have discussed this and determined that adding the two missing authors is the best solution. The two new authors are:

Neha Shah

Department of Physical Medicine and Rehabilitation, Northwestern Medicine at Marianjoy Rehabilitation Hospital, Wheaton, USA

\section{Prempreet Bajaj}

Department of Orthopedic Surgery and Rehabilitation, Loyola University Medical Center, Maywood, USA

\section{Abstract}

Osteoarthritis is a prominent cause of disability in older adults, especially with an increasingly obese and aging population. Clinical management of pain secondary to osteoarthritis should focus on education and self-management with exercise and weight management as a priority. Surgical intervention should only be considered once conservative measures have failed. This review provides a clinical update on the pathogenesis, diagnosis, and management of osteoarthritis.

Categories: Pain Management, Preventive Medicine, Orthopedics

Keywords: osteoarthritis, degenerative joint disease, pain management, sports medicine, non-surgical, orthopedics, preventative medicine, exercise, radiofrequency ablation

\section{Introduction And Background}

Osteoarthritis is a degenerative joint disease involving the cartilage and surrounding tissues of a joint. The cartilage itself is devoid of innervation and not the source of pain associated with osteoarthritis. Rather the wearing away of the cartilage exposes the highly innervated periosteum to damage, and this leads to the perception of "joint pain" [1]. Osteoarthritis is the most common form of arthritis and a leading cause of disability and debility in the United States [2]. Disease progression is generally slow, and over time it causes damage to the articular cartilage, remodeling of bone, ligament laxity, and osteophyte formation to name a few [3]. As such, osteoarthritis can be thought of as a disease of the whole joint. Pain is often the chief complaint and dominant symptom of osteoarthritis, and there is evidence that it should be addressed within a biopsychosocial model that is individualized to the patient [4].

Osteoarthritis also represents an extremely large health burden with significant implications not only in health care but socioeconomically as well [5]. Increasing obesity levels combined with a growing aging population globally has caused osteoarthritis to be a significant and growing issue. Knee osteoarthritis is the most common site of osteoarthritis, followed by the hand and hip, and is found to be present in $19.2 \%-27.8 \%$ of adults above 45 years and $37.4 \%$ of adults above 60 years [6]. This figure is even larger worldwide with a recent global analysis approximating that $85 \%$ of osteoarthritis cases being located in the knee [7]. While increases in life span and body mass index are thought to contribute to the significant rise in knee osteoarthritis in the post-industrial era, those factors alone do not explain this change [8]. Regardless of the cause, the burden of osteoarthritis leads to a decrease in the quality of life. The presence of chronic knee pain has been found to be a strong predictor of future disability and dependency in older adults [9].

There is evidence that among this large and growing population of patients suffering from osteoarthritis, many of them do not receive optimal management [10]. This review will present recent updates in the 


\section{Review}

\section{Assessing the Patient}

Osteoarthritis is a diagnosis made clinically based off of symptoms including pain, functional limitations, and morning stiffness among others. A brief physical examination can reveal many of the appropriate diagnostic criteria such as restricted or painful movement, crepitus, and joint-line tenderness, and is enough to make a diagnosis [11]. It has been shown that the rate of radiographic osteoarthritis is much higher than that of symptomatic arthritis [12], but plain radiographs should be considered if the presentation is atypical or other diagnoses are suspected. Women are more at risk of developing osteoarthritis. Between the ages of 50 and 75 years, the risk for hand and knee osteoarthritis appears to increase much more rapidly in women when compared to men [5]. For knee osteoarthritis, strong risk factors besides age, obesity, and female sex include previous knee injury as well as malalignment of the knee [13] and should be assessed, especially, in patients who were or are athletes. In addition, knee extensor muscle strength should be evaluated as this has also been shown to be a risk factor for knee osteoarthritis [14]. For hip osteoarthritis, the joint should be assessed for deformities such as acetabular dysplasia or cam deformity as this has been shown to be a risk factor [15]. In terms of social risk factors, work that involves heavy lifting has been shown to increase the risk of both hip and knee osteoarthritis, and work that involves a lot of kneeling is associated with increased risk of knee osteoarthritis [16]. Assessment should include a complete history and physical examination, with particular attention given to how symptoms are affecting quality of life, including function, mood, relationships, leisure activities, and sleep as this facilitates shared decision-making, which has been shown to ultimately improve outcomes [17].

The most common chief complaint in patients with osteoarthritis is pain. Other symptoms include crepitus, reduced range of motion, morning stiffness, swelling, muscle weakness, and joint instability [18]. Of note, comorbidities such as cardiovascular disease and diabetes have been shown to be predictive of faster deterioration and progression of pain [19]. The pain in osteoarthritis is typically brought on with weightbearing activity and is as such predictable, especially in early stages. Once the disease progresses the symptoms of pain tend to become more severe and unpredictable, both of which have been shown to be extremely strong indicators of patient's finding their pain unacceptable [20].

X-ray and MRI imaging have been shown to reveal only moderate associations between the presence of pain and structural osteoarthritis [21]. However, this association becomes much stronger in patients with very frequent pain symptoms [22]. Of note, specific findings including synovitis and bone marrow lesions appear to contribute more to pain than overall structural change to pain in osteoarthritis [23].

Increased activity of peripheral nociceptors caused by progressive tissue injury and inflammation in the affected joint is the primary nociceptive pain mechanism in osteoarthritis; however, neuropathic and central pain mechanisms seem to be present in a large proportion of patients with osteoarthritis [24]. Central pain mechanisms include loss of descending antinociceptive pathways as well as enhanced descending pain pathway activity [25]. Neuropathic pain appears to arise from nerve changes in peripheral nervous system or spinal cord as well as structural changes in joint innervation itself [26].

\section{Non-Surgical Management}

Self-management, exercise, weight loss (if overweight or obese), walking aids (if needed), and education are widely accepted as first-line treatment for osteoarthritis and should be initiated as early as possible in the disease process [27]. Patient education should focus on the importance of regular physical activity and exercise as well as appropriate weight management in addition to information about how these conservative measures can potentially avoid surgery and improve quality of life $[28,29]$. Individualized exercise regimens, particularly strength training and aerobic exercises, have been shown to be extremely effective in improving joint motion and decreasing pain [30].

Initial pharmacological management in osteoarthritis includes non-steroidal anti-inflammatory drugs (NSAIDs) and acetaminophen. However, a recent meta-analysis has concluded that acetaminophen is not an effective first-line treatment due to its small area of effect, while oral NSAIDs are effective in terms of clinical improvement of function and pain, although both have safety concerns [31]. Because safety is an important consideration when choosing an appropriate NSAID, it is preferable to restrict usage to only as needed at the smallest effective dose. Of note, a 2018 meta-analysis revealed that topical NSAIDs were effective for pain relief in osteoarthritis with no serious gastrointestinal or renal side effects [32]. In addition, some guidelines advise caregivers to consider duloxetine for pain refractory to initial pharmacological management. It is thought that the anxiolytic effects coupled with central pain inhibition help address neuropathic and central pain mechanisms contributing to osteoarthritis [33]. A metaanalysis published in 2015 concluded that celecoxib, glucosamine, chondroitin, and the combination of glucosamine and chondroitin help relieve joint pain when compared with placebo, but only glucosamine plus chondroitin showed clinically significant improvement from baseline function and therefore should also be considered for non-surgical management [34]. It should be noted, however, that health authorities and 
Patients that do not or no longer respond to oral or topical analgesics should begin intra-articular corticosteroid injections. Patients with evidence of joint inflammation have been shown to respond better to corticosteroid injection than those without, and a large body of evidence suggests that intra-articular corticosteroid injections are much more effective in patients with severe pain compared to those with less pain [27]. Alternatives such as hyaluronic acid injections have been shown to have beneficial effects such as intra-articular lubrication, anti-inflammatory, analgesic, and chondroprotective effects, among others [35]; however, there are inconsistent findings on clinical studies and a high cost associated with this treatment, which has led to the most recent Osteoarthritis Research Society and American College of Rheumatology guidelines to neither recommend nor discourage the use of this treatment [36].

Comparisons between non-opiates and opiates for the relief of pain in osteoarthritis have shown a greater effect with non-opiates as well as increased risk of adverse effects from opiates [37]. This in addition to the high risk of addictive behavior and overdosage has led to opposition to prescribing opioids to patients with osteoarthritis in the medical community [38]. In addition, a 2019 Cochrane Systematic Review showed that tramadol alone and in combination with acetaminophen had no important benefit on pain reduction compared to placebo control [39].

The literature around the innervation of the knee has shown marked variability in the distribution of the nerve supply, and while there is general consensus that the nerves involved are branches of the femoral, sciatic, and obturator, there has been disagreement within the scientific community about exactly which nerves supply the knee capsule and what are the best targets for radiofrequency ablation (RFA) [40,41]. For the purposes of this paper, we will follow the studies by Gardner [40] and Kennedy [42], which are the basis of most anatomy textbooks, and simplify the nerves by splitting them into two groups: an anterior and a posterior group of nerves. The posterior group of afferent nerves originates from the sciatic nerve (primarily its tibial branch) and a variable amount by branches from the posterior branch of the obturator nerve, which form a plexus with the popliteal vessels. The largest and most consistent nerve supplying the knee is the posterior articular branch of the posterior tibial nerve. The tibial nerve remains in the posterior compartment of the leg and gives off the superomedial (SM) and inferomedial (IM) genicular nerves. The posterior group supplies the innervation to the posterior capsule, the external part of the menisci, the anterior and posterior cruciate ligaments, and may reach as far as the infrapatellar (IP) fat pad. The anterior group includes the afferent nerves of the femoral (the branches to vastus medialis, vastus lateralis, and vastus intermedius) and the common peroneal nerve with two articular nerves (the lateral articular and recurrent peroneal nerves) $[40,41]$. The common peroneal splits off the sciatic nerve but passes into the anterior compartment and contributes to the superior lateral (SL) genicular nerve. The anterior group supplies the anteromedial and anterolateral capsules as well as the associated ligaments.

The specific nerves targeted most commonly by radiofrequency neurotomy are the three genicular nerves mentioned: superolateral genicular branch from the common peroneal nerve, the SM, and IM genicular branches from the tibial nerve. The technique for targeting these nerves was popularized by Choi et al. [43] who found significant improvement in pain at three months in the neurotomy patients versus control patients who received a sham procedure with a block. However, despite the popularity of this approach, the anatomical basis and reliability of the bony landmarks described in the study were confusing and created some controversy with the accuracy of the targets being questioned. Part of this was due to a study being performed at the same time by Ikeuchi et al. where they compared RFA of knee sensory nerves at two different sites (the medial retinacular nerve and the IP branch of the saphenous nerve) to a nerve block [44], with similarly positive results at three months.

The result of this complex innervation of the knee has been the development of multiple approaches being described in different studies. In a review of current literature conducted by Jamison and Cohen, they reviewed nine clinical trials which all not only demonstrated significant benefits in reduction of pain and improvement in function but also mentioned the wide range of procedural targets. These targets included the SM, IM, and SL genicular nerves in combination; the saphenous nerve; the sciatic nerve; the IP genicular nerve; the IP and SM genicular nerves in combination; the femoral, tibial, saphenous, and peripatellar plexus in combination; and the intra-articular joint space [45].

On April 13, 2017, the Federal Drug Administration approved cooled RFA as a treatment option for chronic moderate to severe knee pain caused by osteoarthritis. The approval of this approach to treat osteoarthritis pain in the knee was based on a randomized, double-blinded study funded by the device manufacturer (Halyard Health, now known as Avanos, Georgia, USA), which compared the ablation of the three genicular nerves technique (popularized by Choi et al.) via this new delivery system to a single intra-articular steroid injection into the affected knee. This study showed $74.1 \%$ of cooled RFA patients reported greater than $50 \%$ pain relief at six months versus $16.2 \%$ of intra-articular steroid injection patients [46]. Prior to this development, the treatment of pain from late-stage osteoarthritis of the knee was essentially limited to surgery. As a result, in 2003, 402,100 people underwent total knee arthroplasties with a projection of as many as 3.4 million replacements annually by 2030 [47]. However, not all patients are candidates for surgery due to age or comorbidities, and after having failed conservative treatment options such as bracing, physical therapy, medical management, and steroid or hyaluronic acid derivative injections, there were no other 
treatment options. The results of this study were similar to a 2016 study performed by Sari et al., which found genicular nerve radiofrequency neurotomy to be superior to an intra-articular steroid injection into the knee at one and three months [48].

As the use of genicular nerve RFA has expanded, so too has its applications, and we now see it being used for chronic pain that persists after total knee replacement [49]. A 2017 randomized study by McCormick et al. looked at the validity of performing a prognostic block prior to cooled RFA and found no difference in outcomes between patients who had a positive block compared to patients that did not have a block; $58.6 \%$ of nerve block patients and $64 \%$ of non-nerve block patients had at least $50 \%$ improvement in pain at six months [50]. While this again shows significant improvement in pain as a result of RFA, there is still a large percentage of patients that did not have a good response to the procedure. The reasoning for these "failed" procedures is mixed. Choi et al. hypothesized that the patients who did not respond to RFA occurred due to the variable innervation patterns of the knee [43]. The Jamison et al. review examined poor predictors of outcome based primarily on a review of literature from lumbar facet and sacroiliac joint RFA studies. Certain patient characteristics such as opioid use, age > 65 years, higher baseline pain, and history of depression were thought to play a role, as were procedural factors. These procedural factors included the type of RFA used (cooled RFA was found to create a larger lesion that continuous RFA); within the continuous RFA and pulsed RFA groups, larger probes and duration of treatment were found to have larger lesions. The larger lesions were thought to relate to some of the better outcome seen in cooled RFA versus continuous or pulsed RFA, but results have been mixed [45].

\section{Conclusions}

The global population will be confronted with an increasing burden of osteoarthritis in the coming years, and addressing the disease process early in its course will likely vastly improve outcomes. In order to accomplish this, preventive measures beyond promoting weight loss and exercise should be investigated, as well as interventions addressing risk factors for osteoarthritis and specific guidelines for early diagnostic criteria and management. In addition, different phenotypes of pain are an emerging topic that is relevant to osteoarthritis and its management. As our understanding of these phenotypes increase, providers should use this knowledge to individualize treatment for osteoarthritis. Such targeted treatment can help with adherence and prevention of progression of osteoarthritis.

\section{Additional Information \\ Disclosures}

Conflicts of interest: In compliance with the ICMJE uniform disclosure form, all authors declare the following: Payment/services info: All authors have declared that no financial support was received from any organization for the submitted work. Financial relationships: All authors have declared that they have no financial relationships at present or within the previous three years with any organizations that might have an interest in the submitted work. Other relationships: All authors have declared that there are no other relationships or activities that could appear to have influenced the submitted work.

\section{References}

1. Kukreja P, Feinstein J, Kalagara HK, et al.: A summary of the anatomy and current regional anesthesia practices for postoperative pain management in total knee arthroplasty. Cureus. 2018, 10:2755. 10.7759/cureus. 2755

2. Litwic A, Edwards MH, Dennison EM, et al.: Epidemiology and burden of osteoarthritis. Br Med Bull. 2013, 105:185-199. 10.1093/bmb/lds038

3. Neogi T: The epidemiology and impact of pain in osteoarthritis . Osteoarthritis Cartilage. 2013, 21:11451153. 10.1016/j.joca.2013.03.018

4. Prieto-Alhambra D, Judge A, Javaid MK, et al.: Incidence and risk factors for clinically diagnosed knee, hip and hand osteoarthritis: influences of age, gender and osteoarthritis affecting other joints. Ann Rheum Dis. 2014, 73:1659-1664. 10.1136/annrheumdis-2013-203355

5. Lawrence RC, Felson DT, Helmick CG, et al.: Estimates of the prevalence of arthritis and other rheumatic conditions in the United States: Part II. Arthritis Rheum. 2008, 58:26-35. 10.1002/art.23176

6. Vos T, Allen C, Arora M, et al.: Global, regional, and national incidence, prevalence, and years lived with disability for 310 diseases and injuries, 1990-2015: a systematic analysis for the Global Burden of Disease Study 2015. Lancet. 2016, 388:1545-1602. 10.1016/S0140-6736(16)31678-6

7. Wallace IJ, Worthington S, Felson DT, et al.: Knee osteoarthritis has doubled in prevalence since the mid20th century. Proc Natl Acad Sci. 2017, 114:9332-9336. 10.1073/pnas.1703856114

8. Jinks C, Jordan K, Croft P: Osteoarthritis as a public health problem: the impact of developing knee pain on physical function in adults living in the community: (KNEST 3). Rheumatology (Oxford). 2007, 46:877-881. 10.1093/rheumatology/kem013

9. Runciman WB, Hunt TD, Hannaford NA, et al.: CareTrack: assessing the appropriateness of health care delivery in Australia. Med J Aust. 2012, 197:548-549. 10.5694/mja12.10510

10. Altman R, Asch E, Bloch D, et al.: Development of criteria for the classification and reporting of osteoarthritis: classification of osteoarthritis of the knee. Arthritis Rheum. 1986, 29:1039-1049. 10.1002/art.1780290816

11. Pereira D, Peleteiro B, Araújo J, et al.: The effect of osteoarthritis definition on prevalence and incidence estimates: a systematic review. Osteoarthritis Cartilage. 2011, 19:1270-1285. 10.1016/j.joca.2011.08.009 
12. Silverwood V, Blagojevic-Bucknall M, Jinks C, et al.: Current evidence on risk factors for knee osteoarthritis in older adults: a systematic review and meta-analysis. Osteoarthritis Cartilage. 2015, 23:507-515. 10.1016/j.joca.2014.11.019

13. Brouwer GM, van Tol AW, Bergink AP, et al.: Association between valgus and varus alignment and the development and progression of radiographic osteoarthritis of the knee. Arthritis Rheum. 2007, 56:12041211. 10.1002/art.22515

14. Øiestad BE, Juhl CB, Eitzen I, et al.: Knee extensor muscle weakness is a risk factor for development of knee osteoarthritis. a systematic review and meta-analysis. Osteoarthritis Cartilage. 2015, 23:171-177. 10.1016/j.joca.2014.10.008

15. Saberi Hosnijeh F, Kavousi M, Boer CG, et al.: Development of a prediction model for future risk of radiographic hip osteoarthritis. Osteoarthritis Cartilage. 2018, 26:540-546. 10.1016/j.joca.2018.01.015

16. Ezzat AM, Li LC: Occupational physical loading tasks and knee osteoarthritis: a review of the evidence . Physiother Can. 2014, 66:91-107. 10.3138/ptc.2012-45BC

17. Osteoarthritis: care and management. (2014). Accessed: August 18, 2020: https://www.nice.org.uk/guidance/cg177.

18. Hunter DJ, McDougall JJ, Keefe FJ: The symptoms of osteoarthritis and the genesis of pain. Rheum Dis Clin North Am. 2008, 34:623-643. 10.1016/j.rdc.2008.05.004

19. Calders P, Van Ginckel A: Presence of comorbidities and prognosis of clinical symptoms in knee and/or hip osteoarthritis: a systematic review and meta-analysis. Semin Arthritis Rheum. 2018, 47:805-813. 10.1016/j.semarthrit.2017.10.016

20. Liu A, Kendzerska T, Stanaitis I, et al.: The relationship between knee pain characteristics and symptom state acceptability in people with knee osteoarthritis. Osteoarthritis Cartilage. 2014, 22:178-183. 10.1016/j.joca.2013.11.012

21. Bedson J, Croft PR: The discordance between clinical and radiographic knee osteoarthritis: a systematic search and summary of the literature. BMC Musculoskelet Disord. 2008, 9:116. 10.1186/1471-2474-9-116

22. Schiphof D, Oei EH, Hofman A, et al.: Sensitivity and associations with pain and body weight of an MRI definition of knee osteoarthritis compared with radiographic Kellgren and Lawrence criteria: a populationbased study in middle-aged females. Osteoarthritis Cartilage. 2014, 22:440-446. 10.1016/j.joca.2013.12.017

23. Zhang Y, Nevitt M, Niu J, et al.: Fluctuation of knee pain and changes in bone marrow lesions, effusions, and synovitis on magnetic resonance imaging. Arthritis Rheum. 2011, 63:691-699. 10.1002/art.30148

24. Fu K, Robbins SR, McDougall JJ: Osteoarthritis: the genesis of pain. Rheumatology (Oxford). 2018, 57:43-50. 10.1093/rheumatology/kex419

25. Lluch E, Torres R, Nijs J, et al.: Evidence for central sensitization in patients with osteoarthritis pain: a systematic literature review. Eur J Pain. 2014, 18:1367-1375. 10.1002/j.1532-2149.2014.499.x

26. Dimitroulas T, Duarte RV, Behura A, et al.: Neuropathic pain in osteoarthritis: a review of pathophysiological mechanisms and implications for treatment. Semin Arthritis Rheum. 2014, 44:145-154. 10.1016/j.semarthrit.2014.05.011

27. Nelson AE, Allen KD, Golightly YM, et al.: A systematic review of recommendations and guidelines for the management of osteoarthritis: the chronic osteoarthritis management initiative of the U.S. bone and joint initiative. Semin Arthritis Rheum. 2014, 43:701-712. 10.1016/j.semarthrit.2013.11.012

28. French SD, Bennell KL, Nicolson PJA, et al.: What do people with knee or hip osteoarthritis need to know? An international consensus list of essential statements for osteoarthritis. Arthritis Care Res (Hoboken). 2015, 67:809-816. 10.1002/acr.22518

29. Vaishya R, Pariyo GB, Agarwal AK, et al.: Non-operative management of osteoarthritis of the knee joint. J Clin Orthop Trauma. 2016, 7:170-176. 10.1016/j.jcot.2016.05.005

30. Fransen M, McConnell S, Hernandez-Molina G, et al.: Exercise for osteoarthritis of the hip . Cochrane Database Syst Rev. 2014, 4:10.1002/14651858.CD007912.pub2

31. da Costa BR, Reichenbach S, Keller N, et al.: Effectiveness of non-steroidal anti-inflammatory drugs for the treatment of pain in knee and hip osteoarthritis: a network meta-analysis. Lancet. 2017, 390:21-33. 10.1016/S0140-6736(17)31744-0

32. Zeng C, Wei J, Persson MSM, et al.: Relative efficacy and safety of topical non-steroidal anti-inflammatory drugs for osteoarthritis: a systematic review and network meta-analysis of randomised controlled trials and observational studies. Br J Sports Med. 2018, 52:642-650. 10.1136/bjsports-2017-098043

33. Wang ZY, Shi SY, Li SJ, et al.: Efficacy and safety of duloxetine on osteoarthritis knee pain: a meta-analysis of randomized controlled trials. Pain Med. 2015, 16:1373-1385. 10.1111/pme.12800

34. Zeng C, Wei J, Li H, et al.: Effectiveness and safety of glucosamine, chondroitin, the two in combination, or celecoxib in the treatment of osteoarthritis of the knee. Sci Rep. 2015, 5:16827. 10.1038/srep16827

35. Stern R, Jedrzejas MJ: Hyaluronidases: their genomics, structures, and mechanisms of action . Chem Rev. 2006, 106:818-839. 10.1021/cr050247k

36. Bowman S, Awad ME, Hamrick MW, et al.: Recent advances in hyaluronic acid based therapy for osteoarthritis. Clin Transl Med. 2018, 7:6. 10.1186/s40169-017-0180-3

37. Krebs EE, Gravely A, Nugent S, et al.: Effect of opioid vs nonopioid medications on pain-related function in patients with chronic back pain or hip or knee osteoarthritis pain: the SPACE randomized clinical trial. JAMA. 2018, 319:872-882. 10.1001/jama.2018.0899

38. Deveza LA, Hunter DJ, Van Spil WE: Too much opioid, too much harm. Osteoarthritis Cartilage. 2018, 26:293-295. 10.1016/j.joca.2017.12.003

39. Toupin AK, Bisaillon J, Welch V, et al.: Tramadol for osteoarthritis. Cochrane Database Syst Rev. 2019, 5:10.1002/14651858.CD005522.pub3

40. Gardner E: The innervation of the knee joint. Anat Rec. 1948, 101:109-130. 10.1002/ar.1091010111

41. Franco CD, Buvanendran A, Petersohn JD, et al.: Innervation of the anterior capsule of the human knee: implications for radiofrequency ablation. Reg Anesth Pain Med. 2015, 40:363-368. 10.1097/aap.0000000000000269

42. Kennedy JC, Alexander IJ, Hayes KC: Nerve supply of the human knee and its functional importance . Am J Sports Med. 1982, 10:329-335. 10.1177/036354658201000601 


\section{Cureus}

43. Choi WJ, Hwang SJ, Song JG, et al.: Radiofrequency treatment relieves chronic knee osteoarthritis pain: a double-blind randomized controlled trial. Pain. 2011, 152:481-487. 10.1016/j.pain.2010.09.029

44. Ikeuchi M, Ushida T, Izumi M, et al.: Percutaneous radiofrequency treatment for refractory anteromedial pain of osteoarthritic knees. Pain Med. 2011, 12:546-551. 10.1111/j.1526-4637.2011.01086.x

45. Jamison DE, Cohen SP: Radiofrequency techniques to treat chronic knee pain: a comprehensive review of anatomy, effectiveness, treatment parameters, and patient selection. J Pain Res. 2018, 11:1879-1888. 10.2147/jpr.S144633

46. Davis T, Loudermilk E, DePalma M, et al.: Prospective, multicenter, randomized, crossover clinical trial comparing the safety and effectiveness of cooled radiofrequency ablation with corticosteroid injection in the management of knee pain from osteoarthritis. Reg Anesth Pain Med. 2018, 43:84-91. 10.1097/aap.0000000000000690

47. Kurtz S, Ong K, Lau E, et al.: Projections of primary and revision hip and knee arthroplasty in the United States from 2005 to 2030. J Bone Joint Surg Am. 2007, 89:780-785. 10.2106/JBJS.F.00222

48. Sarı S, Aydın ON, Turan Y, et al.: Which one is more effective for the clinical treatment of chronic pain in knee osteoarthritis: radiofrequency neurotomy of the genicular nerves or intra-articular injection?. Int J Rheum Dis. 2018, 21:1772-1778. 10.1111/1756-185X.12925

49. Qudsi-Sinclair S, Borrás-Rubio E, Abellan-Guillén JF, et al.: A comparison of genicular nerve treatment using either radiofrequency or analgesic block with corticosteroid for pain after a total knee arthroplasty: a double-blind, randomized clinical study. Pain Pract. 2017, 17:578-588. 10.1111/papr.12481

50. McCormick ZL, Reddy R, Korn M, et al.: A prospective randomized trial of prognostic genicular nerve blocks to determine the predictive value for the outcome of cooled radiofrequency ablation for chronic knee pain due to osteoarthritis. Pain Med. 2018, 19:1628-1638. 10.1093/pm/pnx286 